\title{
Untersuchungen über die Wechselwirkung von Aromaten mit Antimontrichlorid, III Schmelzdiagramme der binären Systeme*
}

\author{
Investigations about the Interaction of Aromatic Compounds with Antimonychloride, III \\ Melting Diagrams of Binary Systems*
}

\author{
H.-H. Perkampus und E. Schönberger** \\ Institut für Physikalische Chemie der Universität Düsseldorf
}

(Z. Naturforsch. 31 b, 475-479 [1976]; eingegangen am 8. Dezember 1975)

Melting Diagrams, Molecular Compounds, Aromatic Compounds, Antimonytrichloride

This paper presents the results of investigations of the melting diagrams for the systems durene $/ \mathrm{SbCl}_{3}$, pentamethylbenzene/ $\mathrm{SbCl}_{3}$, hexamethylbenzene $/ \mathrm{SbCl}_{3}$, diphenyl $/ \mathrm{SbCl}_{3}$, naphthalene/ $/ \mathrm{SbCl}_{3}$, 2,3-dimethylnaphthalene $/ \mathrm{SbCl}_{3}$, phenanthrene/ $/ \mathrm{SbCl}_{3}$ and anthracene/ $\mathrm{SbCl}_{3}$; in all cases solid complexes with the composition $\mathrm{ArH} \cdot 2 \mathrm{SbCl}_{3}$ were formed.

\section{Einleitung}

Innerhalb unserer Untersuchungen über die Wechselwirkung von Metallhalogeniden mit $\pi$ Elektronensystemen hatten wir uns auch mit dem System Aromat/ $/ \mathrm{SbCl}_{3}$ befaßt1, 2 . Die festen Komplexe, die sich zwischen diesen Komponenten in einem stöchiometrischen Verhältnis $\mathrm{ArH}: \mathrm{SbCl}_{3}=$ 1:1 bzw. 1:2 ausbilden, wurden erstmals von SмIтн $^{3} 1879$ beschrieben und später ausführlicher von Menshutkns mit Hilfe der thermischen Analyse untersucht ${ }^{4}$. Obwohl somit diese Molekülverbindungen schon sehr lange bekannt sind, weiß man bis heute, von einigen Ausnahmen abgesehen, sehr wenig über die Struktur und insbesondere über die Wechselwirkung zwischen der aromatischen Komponente und dem Antimontrichlorid. In den erwähnten Arbeiten finden sich auch Hinweise, daß diese Komplexe z.T. tief gefärbt sind.

In der vorliegenden Arbeit soll über die Ergebnisse der Untersuchung der Schmelzdiagramme

* Teil I u. II s. 1. c. ${ }^{1,2}$.

** E. Schönberger, Auszug aus der Dissertation, Universität Düsseldorf 1975.

Sonderdruckanforderungen an Prof. Dr. H.-H. Perkampus, Universität Düsseldorf, Institut für Physikal. Chemie, D-4000 Düsseldorf, Universitätsstr. 1, Gebäude 2643. einiger Systeme berichtet werden, die bisher in der Literatur nicht beschrieben worden und deren Kenntnis für weiterführende Untersuchungen erforderlich sind. Es handelt sich um die Systeme 1.2.4.5-Tetramethylbenzol/SbCl $\mathrm{Sb}_{3}$, Pentamethylbenzol/ $/ \mathrm{SbCl}_{3}$, Hexamethylbenzol/SbCl 3 , Diphenyl/ $\mathrm{SbCl}_{3}$, Naphthalin/ $\mathrm{SbCl}_{3}, 2.3$-Dimethylnaphthalin/ $\mathrm{SbCl}_{3}$, Penanthren $/ \mathrm{SbCl}_{3}$ und Anthracen/ $/ \mathrm{SbCl}_{3}$.

\section{Experimenteller Teil}

Für die Bestimmung der Abkühlungskurven wurde ein Glasgefäß benutzt, das mit nachgereinigtem Stickstoff gespült werden konnte. Diese Maßnahme ist sehr wesentlich, da bei Gegenwart von Sauerstoff das System Aromat/ $/ \mathrm{SbCl}_{3}$ Nebenreaktionen eingehen kann. Die Substanzen wurden durch einen Normschliff NS 14,5 eingebracht, der gleichzeitig das Thermoelement aufnahm. Die Erwärmung der Proben wurde mit einem heizbaren Magnetrührer vorgenommen. Die gesamte Apparatur war nach außen wärmeisoliert, damit die Abkühlung langsam erfolgte, um die Abkühlungskurven mit ausreichender Genauigkeit auf einem Schreiber registrieren zu können. Als Thermoelement wurden ein KupferKonstantan- und ein Eisen-Konstantan-Thermoelement benutzt, die mit bekannten Schmelzpunkten geeicht worden waren. $\mathrm{SbCl}_{3}$ p. a. der Fa. Riedel de Haen war zur Entfernung von $\mathrm{SbCl}_{5}$ über Antimonmetall im Vakuum destilliert worden. Die Aromaten waren durch Umkristallisieren gereinigt worden. 


\section{Ergebnisse}

\subsection{Das System Durol $/ \mathrm{SbCl}_{3}$}

Das Schmelzdiagramm dieses Systems ist in Abb. 1 wiedergegeben. Es zeichnet sich durch ein stark ausgeprägtes Dystektikum aus, das einer Molekülverbindung im Verhältnis 1:2 entspricht mit einem Schmelzpunkt von $104^{\circ} \mathrm{C}$, der über dem der Komponenten liegt. Dies legt den Schluß nahe, daß die Molekülverbindung relativ stabil ist.

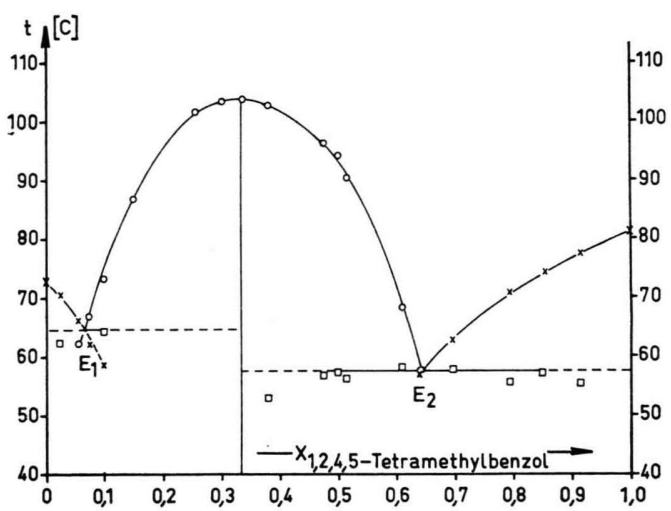

Abb. 1. Schmelzdiagramm des Systems Durol/ $/ \mathrm{SbCl}_{3}$.

\subsection{Das System Pentamethylbenzol/SbCl 3}

In Abb. 2 ist das Schmelzdiagramm des Systems Pentamethylbenzol/ $/ \mathrm{SbCl}_{3}$ wiedergegeben. Im Gegensatz zu dem Diagramm des Systems Durol/ $/ \mathrm{SbCl}_{3}$ in Abb. 1 ist dieses recht kompliziert. Es besitzt ein Dystektikum bei 33,3 Mol\% Pentamethylbenzol (PMB), welches einer kongruent bei $149{ }^{\circ} \mathrm{C}$ schmelzenden Molekülverbindung $\mathrm{PMB} \cdot 2 \mathrm{SbCl}_{3}$ entspricht. Die Soliduskurve der Molekülverbindung gibt Kurve II wieder. Auf der linken Seite gibt Kurve $\mathrm{V}$ die Erstarrung des $\mathrm{SbCl}_{3}$ wieder. Sie zeichnet sich durch das Fehlen eines Eutektikums aus. Die Kurven II und IV schließen eine Mischungslücke zwischen geschmolzener Molekülverbindung und flüssigem $\mathrm{SbCl}_{3}$ ein. Oberhalb der Kurve IV löst sich der Komplex im geschmolzenen $\mathrm{SbCl}_{3}$. Die Mischungslücke reicht bis an die linke Ordinate heran, d.h. die Kurve IV führt bis zum Schmelzpunkt des reinen $\mathrm{SbCl}_{3}$. Die vollständige Unlöslichkeit der Molekülverbindung im $\mathrm{SbCl}_{3}$ erklärt auch das Fehlen eines Eutektikums, da der Schmelzpunkt des $\mathrm{SbCl}_{3}$ nicht erniedrigt wird.

Unterhalb der Erstarrungskurve II der Molekülverbindung liegt ein weiterer Kurvenzug III, der eine Umwandlung in der Schmelze anzeigt. Ober-

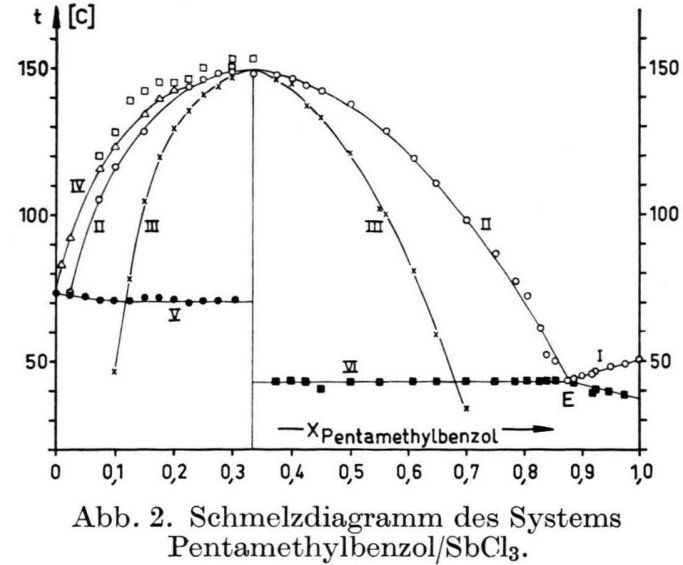

halb der Kurven IV und II sind in Abb. 2 noch einige Meßpunkte als Quadrate eingezeichnet. Sie stammen aus Temperatur-Zeit-Kurven bei einer Aufheizung der Gemische und geben Knickpunkte wieder, nachdem die Molekülverbindung bereits geschmolzen ist. Es dürfte sich hierbei um den Zerfall der Molekülverbindung in die Komponenten handeln. Man kann erkennen, daß alle diese Punkte nur wenige Grade über dem jeweiligen Schmelzpunkt der Molekülverbindung liegen. Danach ist anzunehmen, daß die Beständigkeit des Komplexes stark von der Temperatur abhängt, was auch aus IRspektroskopischen Untersuchungen hervorging ${ }^{2}$.

\subsection{Das System Hexamethylbenzol/ $\mathrm{SbCl}_{3}$}

Das Schmelzdiagramm dieses Systems ist in Abb. 3 wiedergegeben. Es ist ebenfalls sehr kompli-

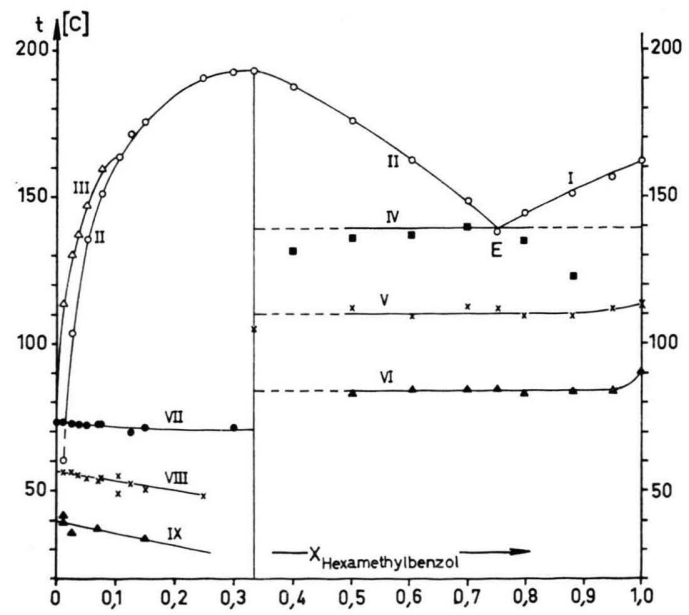

Abb. 3. Schmelzdiagramm des Systems Hexamethylbenzol $/ \mathrm{SbCl}_{3}$. 
ziert und weist insgesamt neun Kurvenzüge auf, deren Meßpunkte bei der Aufnahme der Abkühlungsund Erwärmungskurven erhalten worden sind. Das Schmelzdiagramm (Kurven I, II, IV) zeigt bei einem Gehalt von 33,3 Mol\% HMB ein Dystektikum, welches einer kongruent bei $192,5^{\circ} \mathrm{C}$ schmelzenden Verbindung $\mathrm{HMB} \cdot 2 \mathrm{SbCl}_{3}$ entspricht.

In der erstarrten festen Phase aus HMB und der Molekülverbindung lassen sich zwei weitere Umwandlungen nachweisen (Abb. 3, Kurven V und VI). Erstere (V) dürfte auf die Kristallstrukturänderung des HMB zurückzuführen sein 5,6, wofür eine Umwandlungstemperatur von $115^{\circ} \mathrm{C}$ angegeben wird. Die beiden Umwandlungen sind nur im Bereich von 33,3 bis $100 \mathrm{Mol} \%$ HMB festzustellen. Sie können damit dem Aromaten zugeordnet werden.

Auf der linken Seite des Dystektikums in Abb. 3 liegt zwischen den Kurven II und III wie beim Pentamethylbenzol eine Mischungslücke vor. Daher weist die Erstarrungskurve des $\mathrm{SbCl}_{3}$ (VII) auch keine Gefrierpunktsdepression und somit auch keinen eutektischen Punkt mit der Molekülverbindung auf.

Die Umwandlungspunkte, die den Linien VIII und IX im Schmelzdiagramm entsprechen, dürften den Mischungen aus $\mathrm{SbCl}_{3}$ und Molekülverbindung zuzuschreiben sein, da bei der Erstarrung der reinen Molekülverbindung solche Punkte nicht beobachtet wurden. Eine mögliche Erklärung durch die Änderung der Kristallstruktur des $\mathrm{SbCl}_{3}$, die in einer reinen Probe bei 63,5 und $70^{\circ} \mathrm{C}$ stattfinden soll', dürfte auszuschließen sein, da die Differenzen gegenüber den gemessenen Temperaturen von ungefähr 36 und $55{ }^{\circ} \mathrm{C}$ zu groß sind und reines $\mathrm{SbCl}_{3}$ bei diesen Messungen diese Effekte nicht zeigt. Die Punkte der Linie IX wurden meistens in der unterkühlten Schmelze gefunden und die der Linie VIII hauptsächlich in der festen Phase, nachdem auch das $\mathrm{SbCl}_{3}$ auskristallisiert war.

\subsection{Das System Diphenyl/ $\mathrm{SbCl}_{3}$}

Das Schmelzdiagramm des Systems Diphenyl/ $\mathrm{SbCl}_{3}$ ist in Abb. 4 dargestellt. Das Diagramm zeigt die Bildung einer kongruent bei $72{ }^{\circ} \mathrm{C}$ schmelzenden Molekülverbindung Diphenyl $\cdot 2 \mathrm{SbCl}_{3}$ an. Das Maximum ist ziemlich flach, woraus zu schließen ist, daß eine Molekülverbindung mit schwachen Bindungskräften zwischen den Komponenten gebildet wird.

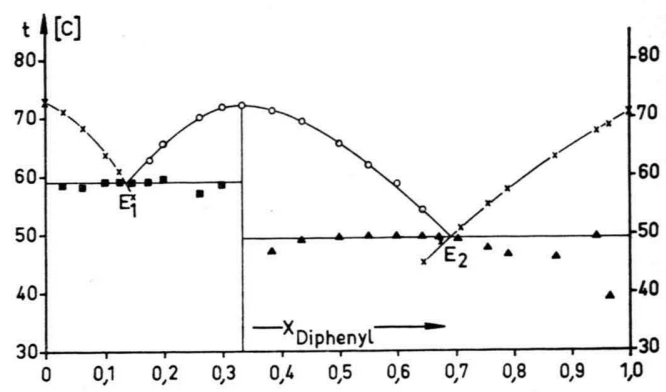

Abb. 4. Schmelzdiagramm des Systems Diphenyl $/ \mathrm{SbCl}_{3}$.

\subsection{Das System Naphthalin $/ \mathrm{SbCl}_{3}$}

Das Schmelzdiagramm des Systems Naphthalin/ $\mathrm{SbCl}_{3}$ ist aus der Litęratur bekannt4,8. Um für spätere Untersuchungen den genauen Verlauf der Umwandlungskurven, besonders unterhalb der Soliduslinie und der Erstarrungskurven bei verschiedenen Konzentrationen interpretieren zu können, wurde dieses System erneut aufgenommen, zumal über diese Molekülverbindung der Zusammensetzung Naphthalin $\cdot 2 \mathrm{SbCl}_{3}$ in der Literatur die meisten Angaben zu finden sind 3,4,8,9. Das ermittelte Schmelzdiagramm zeigt ein typisches ZweiKomponenten-System mit Verbindungsbildung ohne Komplikationen. Der Schmelzpunkt der Molekülverbindung liegt etwas über denen der Komponenten.

\subsection{Das System 2.3-Dimethylnaphthalin $/ \mathrm{SbCl}_{3}$}

Das Schmelzdiagramm dieses Systems zeigt ein flaches Dystektikum bei der Zusammensetzung Aromat: $2 \mathrm{SbCl}_{3}$, welches weit unterhalb der Schmelzpunkte der Komponenten liegt. Es ist daher anzunehmen, daß die Molekülverbindung, die im Verhältnis 1:2 aus Aromat und $\mathrm{SbCl}_{3}$ zusammengesetzt ist, weniger stabil ist. Da dieses Schmelzdiagramm keine Komplikationen aufwies, wurde auf seine Wiedergabe verzichtet.

\subsection{Das System Phenanthren $/ \mathrm{SbCl}_{3}$}

Das Phenanthren reiht sich ebenfalls in die Gruppe der Aromaten ein, die mit $\mathrm{SbCl}_{3}$ eine Verbindung im Verhältnis $1: 2$ bilden. Das Schmelzdiagramm dieses Systems ist in Abb. 5 wiedergegeben. Das Dystektikum ist gut ausgeprägt und besitzt mit $104,5^{\circ} \mathrm{C}$ einen Schmelzpunkt, der $4,5^{\circ}$ höher liegt als der des Phenanthrens. Die Molekülverbindung scheint also relativ stabil zu sein. 


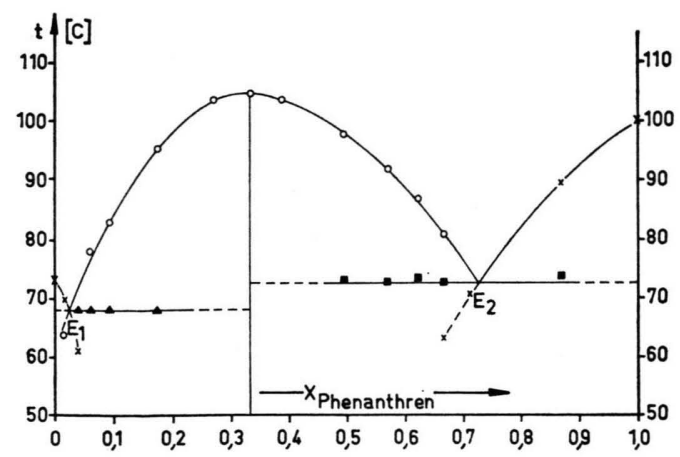

Abb. 5. Schmelzdiagramm des Systems Phenanthren/ $\mathrm{SbCl}_{3}$.

\subsection{Das System Anthracen $/ \mathrm{SbCl}_{3}$}

Abb. 6 gibt das Schmelzdiagramm des Systems Anthracen $/ \mathrm{SbCl}_{3}$ wieder. Das Dystektikum bei einem Molenbruch an Anthracen von 0,333 zeigt eine Molekülverbindung Anthracen $\cdot 2 \mathrm{SbCl}_{3}$ an. Die Molekülverbindung schmilzt zwar kongruent, aber das zweite Eutektikum $\mathrm{E}_{2}$ liegt sehr nahe am rela-

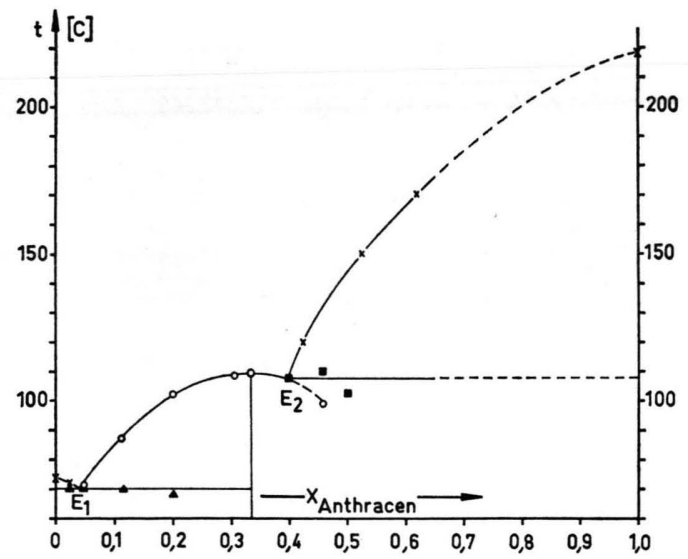

Abb. 6. Schmelzdiagramm des Systems Anthracen $/ \mathrm{SbCl}_{3}$. tiven Maximum der Soliduslinie. Die Molekülverbindung dürfte also nicht sehr stabil sein, zumal auch der Schmelzpunkt der Verbindung weit unter dem des Anthracens liegt. Die Löslichkeit der Molekülverbindung im flüssigen $\mathrm{SbCl}_{3}$ ist sehr gering ${ }^{10}$. Im geschmolzenen Zustand sind $\mathrm{SbCl}_{3}$ und die Molekülverbindung jedoch unbegrenzt mischbar.

Die Aufnahme dieses Schmelzdiagrammes stieß auf Schwierigkeiten, da bei höheren Temperaturen das $\mathrm{SbCl}_{3}$ den Aromaten angreift und schwarze Zersetzungsprodukte entstehen, die sowohl eine Gefrierpunktsdepression als auch eine Unterkühlung der Schmelzen unter die Erstarrungspunkte bewirken. Die gefundenen Werte konnten jedoch gut auf den Schmelzpunkt des Anthracens extrapoliert werden, der sich ohne Schwierigkeiten bestimmen ließ.

\section{Diskussion der Schmelzdiagramme}

In Tab. I sind die charakteristischen Daten der in dieser Arbeit untersuchten Systeme zusammengefaßt. $\mathrm{E}_{1}$ bedeutet das Eutektikum zwischen Dystektikum und $\mathrm{SbCl}_{3} . \mathrm{E}_{2}$ entsprechend zwischen Dystektikum und Aromaten.

Die kryoskopischen Messungen ergaben, daß alle vermessenen Molekülverbindungen im Verhältnis 1:2 aus Aromat und $\mathrm{SbCl}_{3}$ zusammengesetzt sind. Aus einem Schmelzdiagramm läßt sich aber nur das relative Verhältnis und nicht die absolute Zusammensetzung bestimmen.

Hulme und Szymanski ${ }^{9}$ untersuchten die Molekülverbindung Naphthalin $\cdot 2 \mathrm{SbCl}_{3}$ mit Hilfe einer Röntgenstruktur-Analyse.

DeMaldÉ et al. ${ }^{11}$ haben eine Röntgenstrukturuntersuchung der entsprechenden Molekülverbindung Phenanthren $\cdot 2 \mathrm{SbCl}_{3}$ durchgeführt. Die beiden Arbeitskreise stellten fest, daß die Molekül-

Tab. I. Charakteristische Daten der untersuchten Systeme.

\begin{tabular}{lccccccc}
\hline & \multicolumn{2}{c}{ Schmelzpunkt ${ }^{\circ} \mathrm{C}$} & \multicolumn{2}{c}{$\mathrm{E}_{1}$} & \multicolumn{2}{c}{$\mathrm{E}_{2}$} & $\mathrm{ArH}: \mathrm{SbCl}_{3}$ \\
& Aromat & Komplex & $\mathrm{x}_{\text {ArH }}$ & Schmp. ${ }^{\circ} \mathrm{C}$ & $\mathrm{x}_{\text {ArH }}$ & Schmp. ${ }^{\circ} \mathrm{C}$ & \\
\hline Durol & 81,1 & 104 & 0,07 & 64,2 & 0,64 & 57,5 & $1: 2$ \\
Pentamethylbenzol & 50,5 & 149 & - & - & 0,88 & 41,5 & $1: 2$ \\
Hexamethylbenzol & 161,5 & 192,5 & - & - & 0,75 & 139,0 & $1: 2$ \\
Diphenyl & 70,7 & 72,0 & 0,135 & 59,0 & 0,69 & 49,0 & $1: 2$ \\
Naphthalin & 80,2 & 88,1 & 0,091 & 65,2 & 0,70 & 57,0 & $1: 2$ \\
2.3-Dimethylnaphthalin & 105,0 & 59,0 & 0,18 & 51,4 & 0,50 & 49,0 & $1: 2$ \\
Phenanthren & 100,0 & 104,5 & 0,025 & 68,0 & 0,73 & 72,5 & $1: 2$ \\
Anthracen & 218,0 & 109,0 & 0,05 & 70,0 & 0,40 & 107,5 & $1: 2$ \\
\hline
\end{tabular}


verbindung aus einem Molekül Aromat und zwei Molekülen $\mathrm{SbCl}_{3}$ zusammengesetzt sind. Eine neuere Röntgenstrukturuntersuchung von Моотz und LIPKA $^{12}$ bestätigt dies auch für den Komplex Diphenyl $\cdot 2 \mathrm{SbCl}_{3}$. Es ist daher anzunehmen, daß auch die anderen in dieser Arbeit vermessenen Menshutkin-Komplexe die gleiche Zusammensetzung besitzen.

Die Beständigkeit der Molekülverbindungen am Schmelzpunkt ist unterschiedlich, wie aus ihren Schmelzpunkten und der Kurvenform am Dystektikum zu erkennen ist. Sie variiert von der stabilen Molekülverbindung Pentamethylbenzol $\cdot 2 \mathrm{SbCl}_{3}$ mit einem Dystektikum, das oberhalb der Schmelzpunkte der Komponenten liegt, über Anthracen • $2 \mathrm{SbCl}_{3}$, dessen Maximum gerade noch zu erkennen ist, bis zur schwachen Molekülverbindung 2.3Dimethylnaphthalin $\cdot 2 \mathrm{SbCl}_{3}$, die einen Schmelzpunkt unterhalb der Schmelzpunkte beider Komponenten aufweist.

Aus den thermischen Analysen geht hervor, daß die Schmelzen des $\mathrm{SbCl}_{3}$ leicht zur Unterkühlung neigen und daß dieser Effekt um so größer ist, je stärker die „Verunreinigung“ ist, die jeweiligen Molekülverbindungen als Verunreinigungen inbegriffen. Selten jedoch können die Systeme unter den Schmelzpunkt der Aromaten unterkühlt werden.

Dagegen ist eine Unterkühlung der Schmelzen der Molekülverbindung möglich. Dabei ist es unwesentlich, ob die Molekülverbindung als erste Substanz oder nach einer Komponente im eutektischen Ge-

1 H.-H. Perkampus u. E. Baumgarten, Z. Phys. Chemie N.F. 39, 1 [1963].

2 E. Baumgarten, H.-H. Perkampus u. G. Weigelt, Spectrochim. Acta 31 A, 1159 [1975].

3 W. Smith, J. Chem. Soc. 35, 309 [1879]; W. Sмiтн u. G. W. Davin, J. Chem. Soc. 41, 411 [1882].

4 B. Menshutkin, J. Russ. Phys. Chem. Ges. 43, $1275,1303,1329,1805$ [1911]; 44, 1079 [1912] J. Chim. Phys. 9, 314 [1911].

5 Landolt-Börnstein, Neue Serie Bd. III/5a, S. 677 Springer-Verlag, Berlin, Göttingen, Heidelberg 1971. misch auskristallisiert. Die Erfahrung hat gezeigt, daß der Grad der Unterkühlung auch von der Stabilität der Molekülverbindung abhängig ist. Diejenigen Systeme, die ein flaches Dystektikum im Phasendiagramm besitzen und damit instabilere Molekülverbindungen andeuten, neigen zu stärkeren Unterkühlungen.

Diese Beobachtungen lassen sich durch den Aufbau der Molekülverbindung erklären. Als Beispiel kann die Verbindung Phenanthren $\cdot 2 \mathrm{SbCl}_{3}$ betrachtet werden. Der Aufbau dieses Komplexes ist aufgrund einer Röntgenstrukturuntersuchung ${ }^{11}$ bekannt. Die Moleküle des Komplexes bilden im festen Zustand ein Schichtengitter. In ähnlichen Schichten kristallisieren aber auch die Aromaten, so daß man sich einen Kristall der Molekülverbindung hergestellt denken kann, indem $\mathrm{SbCl}_{3}$-Moleküle zwischen die Schichten des Aromaten eingelagert werden. Daher kann sich dieser Aufbau, wenn auch gestört, aus festem Aromat und flüssigem $\mathrm{SbCl}_{3}$ bilden, nicht jedoch, wenn der Aromat schon geschmolzen ist. Es ist zu vermuten, daß Kristallisationskeime so lange vorhanden sind, wie die Schichtenstruktur des Aromaten noch teilweise existiert.

Die vorliegende Arbeit wurde durch Mittel des Verbandes der chemischen Industrie - Fonds der Chemie - unterstützt, wofür wir an dieser Stelle herzlich danken. Der eine von uns (E. ScH.) dankt außerdem für die Gewährung eines Graduiertenstipendiums.

6 G. v. Kimmusheva u. L. P. Yatsenko, Opt. Spectrosc. 33, 49 [1972].

7 W. M. C. Julien u. H. Gerding, J. Roy. Nether. Chem. Soc. 91, 743 [1972].

8 C. Shinomiya, Bull. Chem. Soc. Jap. 41, 61 [1968].

9 R. Hulme u. J. T. Szymanski, Acta Crystallogr. A 25, 753 [1969].

10 l.c. ${ }^{5}$.

11 A. Demaldé, A. Mangia, M. Nardelli, G. Pelizzi u. M. E. Vidoni Tani, Acta Crystallogr. B 28, 147 [1972].

12 D. Mоотz u. A. Lipka, persönliche Mitteilung. 